\title{
Belgeo
}

Revue belge de géographie

$4 \mid 2003$

The dynamics of metropolisation : from words to territory

\section{Editorial : The dynamics of metropolisation : from words to territory / Dynamiques de métropolisation : du discours au territoire}

\section{Pierre Ginet}

\section{(2) OpenEdition}

\section{Journals}

Édition électronique

URL : http://journals.openedition.org/belgeo/16756

DOI : 10.4000/belgeo.16756

ISSN : 2294-9135

Éditeur :

National Committee of Geography of Belgium, Société Royale Belge de Géographie

Édition imprimée

Date de publication : 30 décembre 2003

Pagination : 343-348

ISSN : 1377-2368

Référence électronique

Pierre Ginet, « Editorial : The dynamics of metropolisation : from words to territory / Dynamiques de métropolisation : du discours au territoire », Belgeo [En ligne], 4 | 2003, mis en ligne le 31 décembre 2003, consulté le 24 septembre 2020. URL : http://journals.openedition.org/belgeo/16756 ; DOI : https://doi.org/10.4000/belgeo.16756

Ce document a été généré automatiquement le 24 septembre 2020.

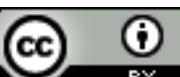

Belgeo est mis à disposition selon les termes de la licence Creative Commons Attribution 4.0 International. 


\title{
Editorial : The dynamics of metropolisation : from words to territory / Dynamiques de métropolisation : du discours au territoire
}

\author{
Pierre Ginet
}

1 Les métropoles sont plongées dans un contexte de concurrence internationale accrue et livrées davantage à elles-mêmes en raison du désengagement des Etats-nations à leur profit pour cause de décentralisation, de fédéralisation et de construction européenne.

2 Ce contexte, assimilable à une crise urbaine, les conduit depuis le début des années 1990 à s'auto-organiser autour de leur propre potentiel de réflexion (les gouvernances métropolitaines) et d'action (formulation de stratégies de développement et de planification innovantes). Ces dynamiques de métropolisation (temps $\mathrm{n}^{\circ} 1 \mathrm{du}$ numéro) font de la métropolisation un processus moteur et auto-entretenu, subdivisable en plusieurs séquences successives. Celles-ci débutent par la structuration de gouvernances métropolitaines et de stratégies spatialisées innovantes. Elles se poursuivent par la déclinaison de ces stratégies sous la forme de projets opérationnels et leur appropriation par la population. L'analyse des effets territoriaux peut être envisagée à différentes échelles. A l'échelle de la tâche urbaine proprement dite (temps $\mathrm{n}^{\circ} 2$ du numéro), marquées par des opérations de renouvellement urbain et la structuration de nouvelles centralités intra-urbaines. A l'échelle d'une région fonctionnelle assimilable à l'aire de métropolisation, où s'expriment sur un mode principalement aréolaire les relations entre centre métropolitain et périphérie métropolitaine (temps $n^{\circ} 3$ du numéro). A l'échelle supra-régionale enfin, champ de recherches encore peu exploré, où se construit puis se définit, au travers de logiques réticulaires, la place des métropoles sur le plan international (temps $\mathrm{n}^{\circ} 4$ du numéro). 
3 L'approche de la métropolisation développée dans ce numéro rend compte de la dimension spatiale de ce processus, au travers de l'inscription territoriale des acteurs qu'elle met en jeu, des espaces stratégiques et de projets de ces derniers, des effets (et absences d'effets) de ces stratégies et projets en termes de recompositions et d'appropriations territoriales. Elle donne une place clef à l'analyse du jeu des acteurs de la métropolisation, de leurs interactions, de leurs rapports de force, de leur inscription et dynamique dans le temps et dans l'espace. Elle fait donc entrer ce numéro de Belgeo dans le domaine d'une géopolitique urbaine en cours d'affirmation.

\section{Éclairages conceptuels}

\section{Métropolisation et territoire, éclairages conceptuels : les nouvelles utopies urbaines}

4 Les utopies urbaines, productions théoriques issues de l'imagination de l'homme, se sont parfois traduites dans la réalité par des réalisations concrètes, avec plus ou moins de succès. Les systèmes théoriques sur lesquels elles se sont fondées ont quant à eux connu une postérité plus longue et servent encore de réservoirs à idées pour les urbanistes contemporains. D. Mons cite Thomas More ou Campanella, qui ont influencé les ingénieurs de la Renaissance; Fourier ou Owen, qui ont à leur manière dicté des solutions formelles en réponse à la ville chaotique de l'industrialisation. Aujourd'hui, la métropolisation, c'est la thèse que l'auteur défend en prenant appui sur plusieurs exemples, correspond autant à la traduction de la mondialisation qu'à une adhésion à des concepts globalisants proches de l'utopie. Trois types de concepts ou de visions stratégiques de la métropole se dégagent : une vision axée sur la réparation de la ville et la protection de son patrimoine, une vision privilégiant le développement urbain durable, une troisième enfin, celle de la "ville générique ».

\section{La production imaginaire d'une « Capitale de l'Europe » : vers la territorialisation d'une identité collective européenne?}

Les manifestations spatiales du système politique européen se sont construites spontanément, sans stratégie symbolique particulière. À l'heure où l'Union Européenne affiche sa volonté de fonder une communauté politique, l'auteur, V. Calay, cherche à analyser l'enjeu symbolique de cette manifestation. Bruxelles est pris comme exemple. L'auteur s'interroge sur la place que Bruxelles peut occuper comme centre symbolique de l'Europe, par analogie avec les systèmes politiques des Etats-nations où un tel processus se produit habituellement. Le tourisme à Bruxelles concourt-il à l'ancrage de la représentation de la capitale belge comme capitale de l'Europe dans l'esprit des personnes qui y séjournent ? L'auteur nuance la réponse apportée à cette question. 


\title{
Métropolisation à l'échelle intra-urbaine : renouvellement urbain et construction de centralités en milieu urbain
}

\author{
Réaffectation urbaine et Développement socio-économique. Le \\ projet « cité de la science et de la connaissance » sur le site de Tour \\ et Taxis à Bruxelles
}

Grands équipements et métropolisation: comment réussir une opération de renouvellement urbain haut de gamme (un "flagship project») sans engager un peu plus Bruxelles dans la spirale de l'inflation des prix du foncier, de la gentrification? Le cas du projet de «Cité de la Science et de la Connaissance » sur l'ancienne friche du site de Tour et Taxis (30 hectares) est analysé par P. Cornut, M. Roelandts, E. Castiau et M.Van Criekingen après que les enseignements d'expériences étrangères aient été synthétisés. Il s'agit d'une opération de haut standing qui doit accroître l'attractivité de Bruxelles et stimuler les investissements étrangers dans des secteurs de pointe. Sur la base d'un patrimoine architectural remarquable, ce projet urbain est axé vers la formation (grande école d'ingénieurs), l'activité économique (attraction d'entreprises innovantes, incubateurs d'entreprises) et culturelle (musée scientifique, centre de congrès). Après avoir montré les risques sociaux de la démarche sur le quartier contigu au site, les auteurs préconisent la mise en oeuvre conjointe de mesures d'accompagnement visant à pérenniser certaines activités économiques dans le tissu économique local et la maîtrise de la polarisation sociale pour éviter tout phénomène d'exclusion et de gentrification.

\section{Services informatiques et centralités métropolitaines en région parisienne}

Ce travail de recherche porte sur le polycentrisme en milieu urbain, observé dans de nombreuses métropoles. J. Fache cherche ici à comprendre le passage d'une structure parisienne dominée par un pôle à une structure polynucléaire à travers une activitétémoin, les services informatiques. Le facteur temps est pris en compte. L'auteur interroge les nouvelles centralités qui émergent pour une activité tertiaire de haut niveau, et relève les discordances qui existent avec la vision communément admise des centralités importantes dans l'agglomération parisienne. C'est aussi la question des causes de l'émergence de ces nouvelles centralités qui est interrogée. L'outil de la diffusion spatiale des services informatiques en région parisienne s'avère être pertinent pour comprendre la formation de cet espace métropolitain polynucléaire. L'article montre que la remise en cause de la centralité principale n'est que relative, et plus que de la dilution globale du centre, il faut sans doute parler d'un glissement réel de certaines fonctionnalités seulement. Une telle logique peut toutefois développer des spécialisations fortes et déséquilibrantes pour une structure polycentrique sans doute trop souvent perçue comme un modèle absolu. 


\title{
Métropolisation à l'échelle régionale : construction d'aires de métropolisation?
}

\author{
Centre et périphéries : Des espaces en compétition ? Enquête sur les \\ types de localisation des entreprises à l'échelle de l'aire \\ métropolitaine bruxelloise
}

8 Dans le contexte de suburbanisation des entreprises de services, la fragmentation politico-administrative de l'aire métropolitaine bruxelloise entre les trois Régions belges crée a priori des situations de concurrence territoriale. L'article de M. Lennert et M. Van Criekingen présente les résultats d'une enquête (menée dans le cadre du projet Comet) sur les types de localisation d'un échantillon d'entreprises de services à la production localisées dans la région fonctionnelle de Bruxelles. L'enquête montre l'existence de plusieurs types de localisation distincts en fonction de l'envergure des firmes, de leur taille, des types de contacts entre prestataires de services et clients ou de leur dynamisme. Elle indique paradoxalement une logique de complémentarité plutôt que de compétition entre espaces dotés de caractéristiques spécifiques au sein de l'ensemble fonctionnel métropolitain bruxellois.

\section{L'intégration des petites villes de l'arc Nord (zone transfrontalière franco-belge) et de l'arc Sud (ex-Pays minier) dans la dynamique métropolitaine lilloise}

9 La métropole lilloise est une conurbation constituée par la réunion de pôles urbains à forte tradition industrielle, marqués par des dynamiques spatiales distinctes. Au nord de la métropole s'étendent par-delà la frontière franco-belge les agglomérations wallonnes de Tournai et Mouscron, et flamande de Courtrai. A vingt kilomètres au sud, en continuité relative avec l'agglomération lilloise, c'est l'ancien bassin minier qui s'étend, selon un arc de cercle englobant les agglomérations de Béthune, Lens, Douai et Valenciennes. P. Ginet montre que la métropolisation en cours tend à rapprocher ces petites villes de l'agglomération lilloise. Un rapprochement qui se traduit par des initiatives stratégiques communes et par la constitution progressive d'une gouvernance élargie. Encore balbutiante, cette dynamique émergente de construction d'une aire de métropolisation grand lilloise est toutefois déjà perceptible.

\section{Métropolisation à l'échelle internationale : logiques de réseaux}

\section{The Global Capacity of Belgium's major cities: Antwerp and Brussels compared}

L'article de B. Derudder et P.-J.Taylor vise deux objectifs : définir une méthode de mesure économique des relations entre les principales villes du monde, fournir une évaluation de la position des deux plus grandes villes belges dans ce réseau urbain global. D'abord, les acteurs montrent que les recherches sur Bruxelles et Anvers ne concernent que très peu leur place respective dans les réseaux de villes internationaux, 
ce qui s'explique en partie par un problème de sources disponibles. Ils proposent ensuite une méthode pour évaluer la place des villes dans des réseaux internationaux. Enfin, ils abordent les cas de Bruxelles et d'Anvers. Si Bruxelles possède une connectivité internationale plus importante qu'Anvers, cette dernière dispose a contrario d'une connectivité locale beaucoup plus intense que Bruxelles. Plus spécifiquement, Bruxelles est particulièrement connectée à l'Extrême Orient asiatique et à l'Amérique du Nord alors que les principaux liens concernant Anvers la relient aux grandes villes européennes voisines (spécialement allemandes).

\section{AUTEUR}

\section{PIERRE GINET}

Guest Editor 